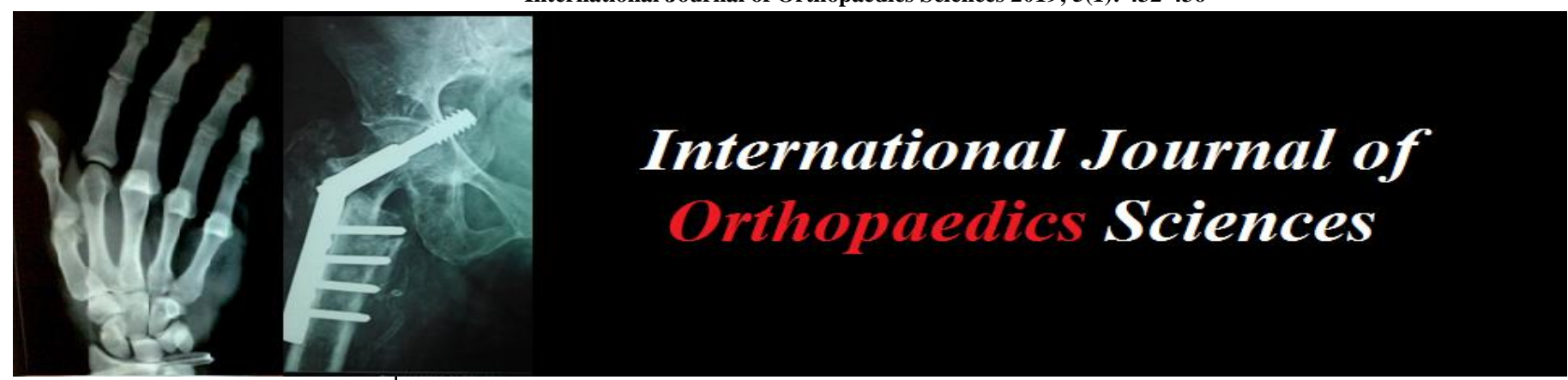

ISSN: $2395-1958$

IJOS 2019; 5(1): 432-436

(C) 2019 IJOS

www.orthopaper.com

Received: 24-11-2018

Accepted: 26-12-2018

Dr. Santosh Kumar Sahu

Assistant Professor of Orthopedics, IMS \& SUM

Hospital Bhubaneswar, SOA

University, Odisha, India

\section{A technique to aid closed reduction in paediatric supracondylar humerus fractures}

\section{Dr. Santosh Kumar Sahu}

DOI: $\underline{\text { https://doi.org/10.22271/ortho.2019.v5.i1h.79 }}$

\section{Abstract}

Anatomic reduction of all displaced pediatric supracondylar humerus fractures is not possible via closed manipulation, thus necessitating open reduction. Open reduction has been associated with increased complications, including elbow stiffness, scarring, iatrogenic neurovascular injury, and longer hospital stays. We used a $3 \mathrm{~mm}$ k-wire to aid in closed reduction thus decreasing the need for conversion to an open procedure, possibly reducing morbidity.

Description of Technique: A percutaneously placed 3-mm k-wire was drilled into the posterior humeral diaphysis and used as a joystick to reduce anterior and posterior, varus and valgus, and rotational deformity. The fracture then was stabilized with1.5/ 2-mm K-wires placed under fluoroscopy and the $3 \mathrm{~mm} \mathrm{k}$-wire was then removed.

Methods: We reviewed all displaced pediatric supracondylar humerus fractures treated at IMS\&SUM Hospital, Bhubaneswar from October 2016 through August 2018.We included 60 fractures meeting criteria for inclusion. These fractures then were divided into two groups. Group A (30 fractures) included fractures treated without implantation of the $3 \mathrm{~mm} \mathrm{~K}$-wire. In this group, if successful reduction could not be achieved via closed manipulations, a formal open reduction was performed. In Group B, (30 fractures) the thick k-wire technique was used to assist with reduction of fractures. All fractures were stabilized with the 1.5/ 2-mm K-wires after the reductions. Injury data, operative technique, complications, and radiographic reduction were analyzed. The minimum followup for both groups was 8 weeks (average 12 weeks; range, 8-40 weeks) to monitor clinical and radiological recovery. Range of motion and loss of carrying angle was assessed according to Flynn's criteria (Table. 8, 9).

Results: No fractures in Group B (zero of 30, 0\%) compared with Group A (three of 30, 10\%) required open reduction. As regards fracture alignment at final followup and postoperative complications, Group B patients had superior results compared to group A.

Conclusions: A posteriorly placed $3 \mathrm{~mm}$ k-wire aids in anatomic reduction and decreases the need for open treatment of displaced pediatric supracondylar humerus fractures, without compromising the complication rate or final radiographic outcome.

Keywords: Pediatric supracondylar humerus fractures, operative technique, fracture alignment

\section{Introduction}

Pediatric supracondylar humerus fractures are the most common fractures around the pediatric elbow ${ }^{[5,6,9]}$. These fractures often are divided into flexion and extension

types, depending on the mechanism of injury and the distal fragment's direction of displacement. Approximately $97 \%$ to $99 \%$ of pediatric supracondylar humerus fractures are extension-type injuries ${ }^{[6,9]}$. Because remodeling is limited [1], meticulous reduction and anatomic alignment are essential to restore normal elbow function and prevent future complications from malreduction and resultant abnormal joint kinematics ${ }^{[6,9,10]}$.

Closed reduction with pin stabilization, introduced by Swenson in $1948^{\text {[12] }}$, is the most common type of treatment for displaced (Gartland Types II and III [6, 9]) pediatric supracondylar humerus fractures ${ }^{[6,12]}$. A Gartland Type II fracture is defined by displacement of the anterior cortex greater than $2 \mathrm{~mm}$, while the posterior cortex remains intact, but it may be hinged. Gartland Type III fractures are completely displaced with no significant cortical contact ${ }^{[6,9]}$. Acceptable reduction is not always attainable through closed manipulation. The median nerve and/or brachial artery may become entrapped in the fracture site, impeding closed reduction ${ }^{[9]}$.

\section{Correspondence}

Dr. Santosh Kumar Sahu Assistant Professor of Orthopedics, IMS \& SUM Hospital Bhubaneswar, SOA University, Odisha, India 
When closed manipulation does not yield anatomic alignment (defined as restoration of the humeral capitellar angle $>9$ on the AP view, intact medial and lateral columns on oblique views, and bisection of the anterior humeral line through the middle third of the capitellum on the lateral view ${ }^{[9]}$ ), a formal open reduction must be performed. The need for open reduction has been reported in $3 \%$ to $15 \%$ of displaced fractures ${ }^{[2,6,8,9]}$. Several authors explored techniques to assist in closed and/or open reduction of the displaced pediatric supracondylar humerus fractures. In 2009, Parmaksizoglu et al. ${ }^{[7]}$ described a method of reduction similar to our reported technique. Their technique involved placing a $\mathrm{K}$-wire from laterally to medially in the humeral diaphysis, "below the deltoid muscle. The K-wire was used as a joystick to aid in manipulation of the humeral diaphysis to achieve fracture reduction with the subsequent stabilization with $\mathrm{K}$-wires. Twenty-two of the 23 pediatric supracondylar humerus fractures in their series had "excellent" or "good" clinical outcomes, according to the criteria of Flynn et al. ${ }^{[4]}$, and no fractures had open reduction ${ }^{[7]}$. However, they did not compare the outcomes with the fractures treated before implementation of this joystick method. The primary differences between the technique described by Parmaksizoglu et al. and ours are the direction and location of pin placement. Their pin is placed from medial to lateral at the level of the deltoid where the radial nerve is medial or posterior at that level. In our technique, the $3 \mathrm{~mm} \mathrm{k}$-wire is placed from posterior to anterior in a more distal location where the radial nerve is lateral. More distal placement allows the pin to be closer to the fracture site and potentially provides more control of the fracture. We describe a novel technique of percutaneous, posterior humeral diaphyseal kwire $(3 \mathrm{~mm})$ placement to aid in the reduction of displaced pediatric supracondylar humerus fractures. We determined whether implementation of the $\mathrm{k}$-wire $(3 \mathrm{~mm})$ technique; (1)decreased the rate of conversion to an open procedure, (2) decreased the operative time and length of hospital stay between formal open reduction and reduction with the k-wire technique, and (3) better final radiographic alignment (4) reduced complications.

Surgical Technique: The indications for surgery were displacement in any plane greater than $2 \mathrm{~mm} /$ Gartland Type II or III. Contraindications were Gartland Type I or nondisplaced fractures. A specific algorithm for number of reduction attempts, time spent doing a closed reduction, exposure time fracture characteristics was followed. The patient was administered general anesthesia and was positioned supine on the operative table. Closed reduction was attempted initially in all Group A patients in the following sequence: (1) axial traction, (2) varus or valgus manipulation to correct coronal displacement, and (3) sagittal alignment was restored with hyperflexion to correct the extension deformity (or hyperextension to correct the rarer flexion deformity). Pronation or supination of the forearm was performed to correct and fine-tune the reduction. A reduction was considered acceptable when the anterior humeral line bisected the middle third of the capitellum as observed on the lateral radiograph and the humeral capitellar angle was normal (range, 9-26) on the AP radiograph ${ }^{[6,9]}$. In Group B patients a $3 \mathrm{~mm}$ k-wire was placed percutaneously in the posterior humeral diaphysis. To place this pin, the patient's shoulder was abducted to 90 and maximally externally rotated (Fig. 3, 4). A 1-cm skin incision was made in the posterior arm and blunt dissection was carried down until the surface of the posterior humeral diaphysis was encountered. The incision was made in the distal third of the humeral shaft to avoid the radial nerve, which is lateral at this location. After the blunt dissection, we inserted the k-wire $(3 \mathrm{~mm})$ through the posterior cortex (Fig. 5, 6). The pin then was advanced through both cortices. The pin was used as a joystick to assist in reduction of the anterior and posterior translation and reduction of varus and valgus malalignment. After fracture stabilization with percutaneous pinning with1.5/ $2 \mathrm{~mm} \mathrm{~K}$-wires, the $3 \mathrm{~mm} \mathrm{k}$-wire was removed.
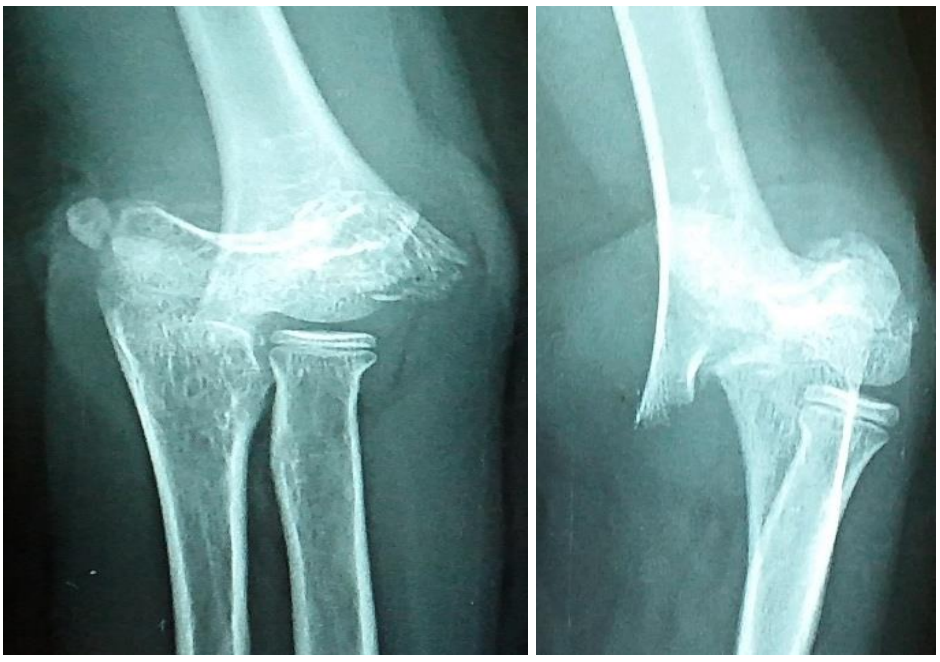

Fig 1-2: (pre-op x-ray shows Gartland Type III fracture with rotational instability.) 

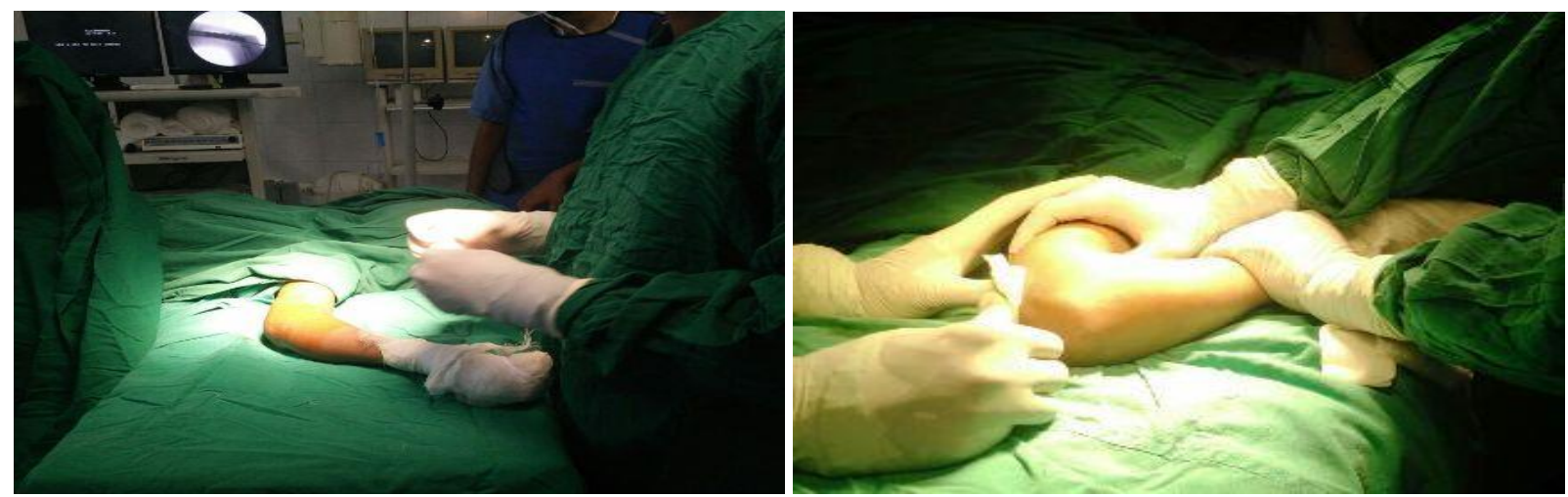

Fig 3-4: (arm is positioned in 90_ abduction and maximal external rotation for pin placement
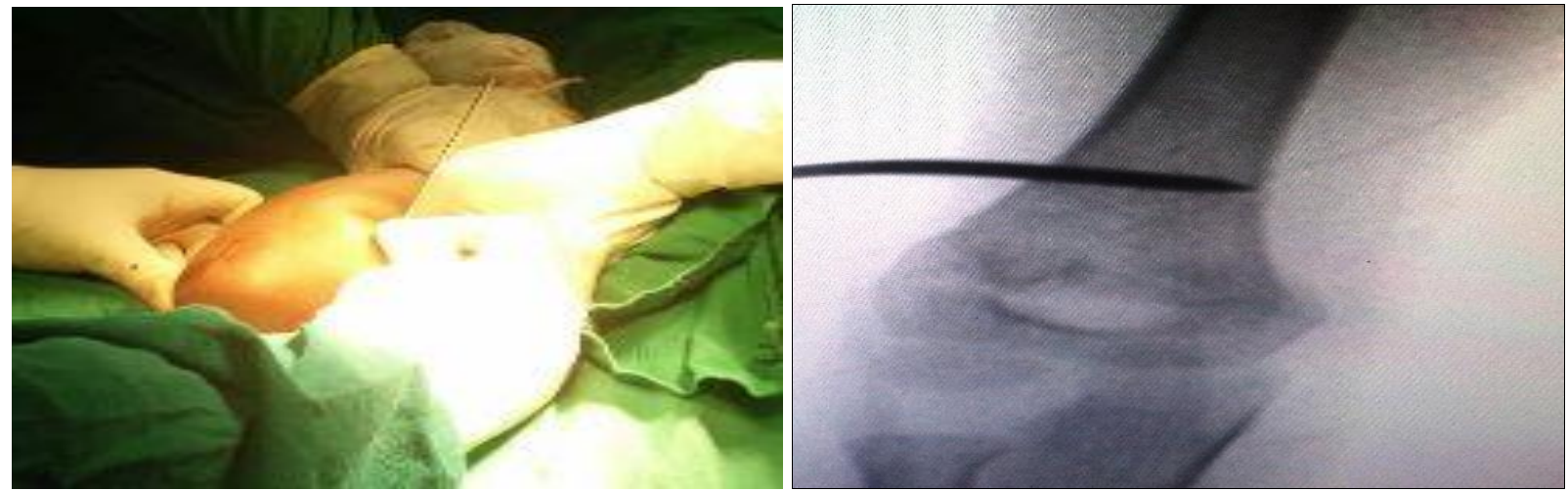

Fig 5-6: (intraoperative and fluoroscopy image shows insertion of the $3 \mathrm{~mm} \mathrm{k}$-wire before its use during reduction.)
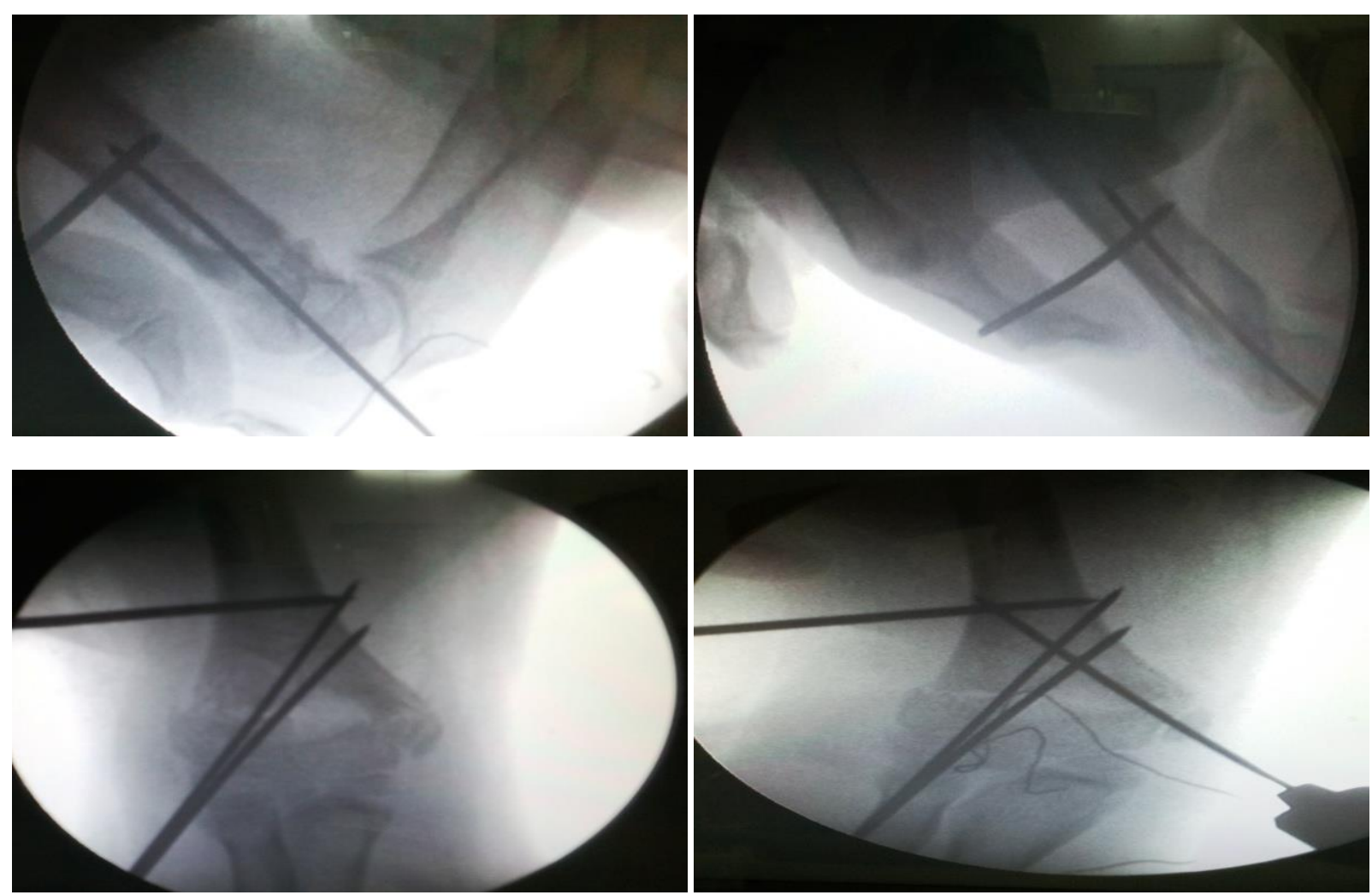

Fig 7-10: (Final fluoroscopy images shows insertion of the $3 \mathrm{~mm} \mathrm{k}$-wire, its use during reduction.)

\section{Material and Methods}

We included 60 displaced (Gartland Types II and III) pediatric supracondylar humerus fractures treated surgically from october 2016 through August 2018 at IMS\&SUM Hospital, Bhubaneswar. The rest 60 patients were divided into two groups depending on whether treatment occurred without (Group A, 30 fractures) or with (Group B, 30 fractures) implementation of the $3 \mathrm{~mm} \mathrm{k}$-wire technique (to aid in closed reduction). The minimum followup for both groups was 8 weeks (average, 12 weeks; range, 8-40 weeks). Each fracture was classified using the Gartland classification for pediatric supracondylar humerus fractures [6]. Neurovascular examinations were performed and recorded. The patients underwent splinting in a comfortable position with the arm placed in a posterior, long arm splint. No closed reduction was attempted in the emergency department. The timing of 
the surgeries was determined by the surgeon and operating room availability. Patients with fractures with associated vascular compromise were taken to the operating room emergently on presentation. After reduction and stabilization, the patients' arms were placed in a bulky dressing and immobilized in a posterior long arm plaster splint or a long arm cast with the elbow flexed to 90 and the forearm in neutral rotation. Operative time, length of stay (admission to discharge) and exposure time were recorded. patients with polytrauma and Patients with concomitant elbow injuries were excluded from the study analysis. Patients were seen for an initial postoperative visit at 1-2weeks. Clinical examination at that time was limited to motor and sensory functions of the hand owing to the cast or splint on the extremity. Anteroposterior and lateral radiographs were obtained to verify maintenance of reduction and alignment. Patients were kept immobilized for 3 to 4 weeks, at which time they were seen in the opd again for repeat clinical examination and radiographs. If bridging callus was seen, the pins were removed and activities were gradually advanced as tolerated. Patients were seen again 4 to 6 weeks after pin removal for repeat radiographs and physical examination. At the final followup measurements of ROM, motor function, and determination of any complications of treatment were done. If subsequent visits revealed motion loss, patients were referred to physical therapy and followed until satisfactory motion was achieved. Anteroposterior and lateral radiographs were evaluated at the final followup to determine final coronal (Fig. 11) and sagittal (Fig. 12) alignment. Humeral capitellar angle, was measured on AP radiographs to determine normal (range, 9-26), varus $(<9)$ or valgus $(>26)$ alignment ${ }^{[6,9]}$. Lateral radiographs were analyzed with reference to the capitellum and its position relative to the anterior humeral line (anterior or posterior). Range of motion and loss of carrying angle was assessed according to Flynn's criteria (Table-8, 9)

Table 1: Age distribution

\begin{tabular}{|c|c|c|}
\hline Group & Number & Age of patients (in yrs) with range \\
\hline Group A & 30 & $5.7(0.6-10.2)$ \\
\hline Group B & 30 & $6.3(1.2-12.0)$ \\
\hline Total & 60 & $5.9(0.6-11.9)$ \\
\hline
\end{tabular}

Table 2: Sex incidence.

\begin{tabular}{|c|c|c|}
\hline Group & Male & Female \\
\hline Group A & $15(50 \%)$ & $15(50 \%)$ \\
\hline Group B & $21(70 \%)$ & $9(30 \%)$ \\
\hline Total & $36(60 \%)$ & $24(40 \%)$ \\
\hline
\end{tabular}

Table 3: Gartland Classification

\begin{tabular}{|c|c|c|}
\hline Group & Type-II & Type-III \\
\hline Group A & $12(40 \%)$ & $18(60 \%)$ \\
\hline Group B & $6(20 \%)$ & $24(80 \%)$ \\
\hline Total & $18(30 \%)$ & $42(70 \%)$ \\
\hline
\end{tabular}

Table 4: (Operative Time)

\begin{tabular}{|c|c|}
\hline Group & Average operative time(in minutes)with range \\
\hline Group A & $45(30-52)$ \\
\hline Group B & $36(25-42)$ \\
\hline
\end{tabular}

Table 5: (No. of cases required open reduction)

\begin{tabular}{|c|c|}
\hline Group & Open reduction \\
\hline Group A & $3(10 \%)$ \\
\hline Group B & $0(0 \%)$ \\
\hline
\end{tabular}

Table 6: (Length of Hospital stay)

\begin{tabular}{|c|c|}
\hline Group & Avg. length of stay (admission to discharge in days) \\
\hline Group A & $1.5(0.9-1.7)$ \\
\hline Group B & $1.2(0.8-1.5)$ \\
\hline
\end{tabular}

Table 7: (Complications)

\begin{tabular}{|c|c|c|c|c|c|c|}
\hline Group & Iatrogenic nerve injury & Compartment syndrome & Vascular injury & Pinsite infection & Deep infection & Malunion \\
\hline Group A & 0 & 0 & 1 & 2 & 0 & 3 \\
\hline Group B & 0 & 0 & 0 & 1 & 0 & 1 \\
\hline
\end{tabular}

Table 8: (Flynn's class range of motion)

\begin{tabular}{|c|c|c|}
\hline Loss of motion in(degrees) & Group A(n=30) & Group B(n=30) \\
\hline Excellent & 22 & 26 \\
\hline Good & 5 & 2 \\
\hline Fair & 0 & 2 \\
\hline Poor & 3 & 0 \\
\hline
\end{tabular}

Table 9: (Flynn's class cosmetic deformity)

\begin{tabular}{|c|c|c|}
\hline $\begin{array}{c}\text { Loss of carrying angle in } \\
\text { (degrees) }\end{array}$ & $\begin{array}{c}\text { Group } \\
\mathbf{A}(\mathbf{n}=\mathbf{3 0})\end{array}$ & $\begin{array}{c}\text { Group } \\
\mathbf{B}(\mathbf{n}=\mathbf{3 0})\end{array}$ \\
\hline Excellent & 23 & 27 \\
\hline Good & 4 & 2 \\
\hline Fair & 0 & 0 \\
\hline Poor & 3 & 1 \\
\hline
\end{tabular}

\section{Results}

Fewer fractures in Group B (one of 30, 3.33\%) compared with Group A (three of 30,10\%) required open reduction. As regards fracture alignment at final followup and postoperative complications, Group B patients had superior results compared to group A. Fractures in Group A had a longer $(\mathrm{p}=$ 0.029) operative time (average, 42 minutes; range, 30-48 minutes), when compared with fractures in Group B (average
36 minutes; range, 25 42minutes) (Table 4). Three (5\%) fractures healed with varus malalignment (two in Group A [6.66\%] and one in Group B [3.33\%]). Lateral radiographs revealed the middle of the capitellum to be anterior to or at the anterior humeral line in $55(91.66 \%)$ fractures (26 in Group A [86.66\%] and 29 in Group B [96.66\%]).There was no difference for individual complications of nerve injury, vascular injury, compartment syndrome or deep infection between Groups A and B (Table 7). Two patients in Group A $(3.7 \%)$ and 1 in group B had superficial pin tract infections. No patients had compartment syndrome, deep infections. All nerve injuries resolved without persistent deficit. One brachial artery injury was noted preoperatively in group A required exploration with the release of constricting bands and the subsequent return of flow. There were no iatrogenic vascular injuries. Evaluation of results by Flynn's lateral approach in surgical treatment of pediatric criteria, in group A, range of motion was regarded as excellent in $22(73 \%)$, good in 5 (17\%) patients, while $26(87 \%)$ excellent, $2(6.6 \%)$ good, $2(6.6 \%)$ fair in group B. $3(10 \%)$ patients in group A and none in group B had poor results as shown in table 8. Flynn's criteria regarding carrying angle, showed statistically significant difference between, two groups. In group A, 23 (76.66\%) patients have excellent and $4(13.33 \%)$ patients 
have good results. In group B, 27 (90\%) were excellent, 2 $(6.6 \%)$ were having good results. Cubitus varus deformity was observed in $1(3.33 \%)$ case of group B, while in $3(10 \%)$ cases in group A. All unsatisfactory or poor results observed in group $\mathrm{A}[3(10 \%)$ cases] and group $\mathrm{B}[1$ case $(3.33 \%)]$ as depicted in Table-9, underwent ORIF.

\section{Discussion}

Pediatric supracondylar humerus fractures are the most common fractures around the pediatric elbow ${ }^{[5,6,9]}$. These fractures often are divided into flexion and extension types, depending on the mechanism of injury and the distal fragment's direction of displacement. Approximately $97 \%$ to $99 \%$ of pediatric supracondylar humerus fractures are extension-type injuries ${ }^{[6,9]}$. Because remodeling is limited ${ }^{[1]}$, meticulous reduction and anatomic alignment are essential to restore normal elbow function and prevent future complications from malreduction and resultant abnormal joint kinematics $[6,9,10]$. Several operative tricks and techniques have been described in the literature, but to our knowledge, no study has been designed to show a decrease in the need for open reduction with these techniques ${ }^{[3,5,7,11]}$. Our technique of k-wire $(3 \mathrm{~mm})$ placement in the posterior humeral diaphysis was associated with a reduction in the conversion rate to an open procedure and in operative time without affecting final radiographic alignment or complications. There are several limitations to the current study. First, we did not have standardized documentation of the preoperative and postoperative physical examinations. Second, we could not measure some possible factors that may have contributed to a decrease in the rate of conversion to an open procedure and/or decreased operative time. This includes the evolving experience of the surgeon regarding closed manipulation and percutaneous pinning techniques. However our findings show that a $3 \mathrm{~mm} \mathrm{k}$-wire placed in the posterior humeral diaphysis can aid in closed reduction of displaced pediatric supracondylar humerus fractures. Furthermore, we found that use of this technique may decrease the need for conversion to an open reduction without compromising the radiographic reduction or clinical outcome.
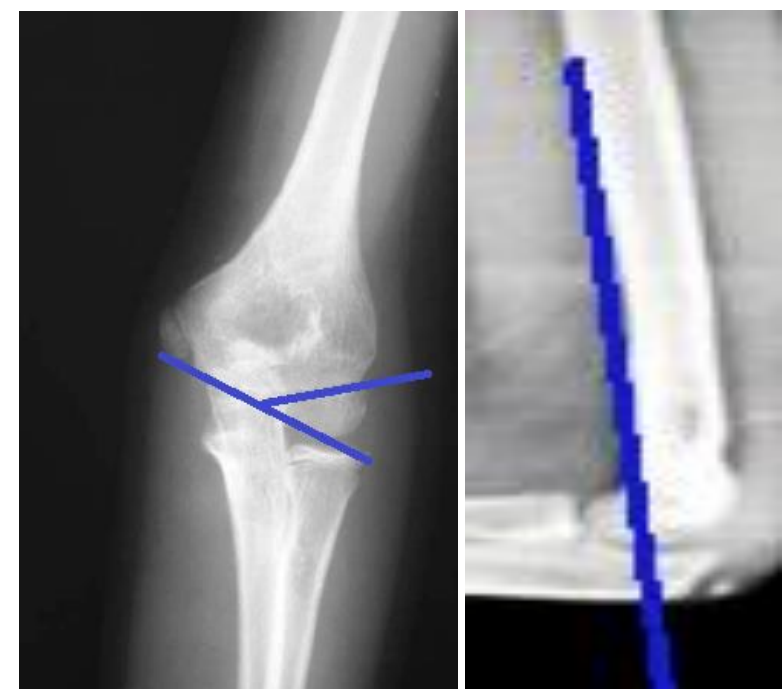

Fig 11, 12: Final ap and lateral radiograph shows acceptable coronal and sagittal alignment with the anterior humeral line, traversing the middle third of the capitellum

\section{References}

1. American Medical Association. CPT1 2012 Professional. Chicago, IL: American Medical Association Press, 2011.
2. Cramer KE, Devito DP, Green NE. Comparison of closed reduction and percutaneous pinning versus open reduction and percutaneous pinning in displaced supracondylar fractures of the humerus in children. $\mathrm{J}$ Orthop Trauma. 1992; 6:407-412.

3. Eberl R, Eder C, Smolle E, Weinberg AM, Hoellwarth $\mathrm{ME}$, Singer G. Iatrogenic ulnar nerve injury after pin fixation and after antegrade nailing of supracondylar humeral fractures in children. Acta Orthop. 2011; 82:606609.

4. Flynn JC, Matthews JG, Benoit RL. Blind pinning of displaced supracondylar fractures of the humerus in children: sixteen years'experience with long-term followup. J Bone Joint Surg Am. 1974; 56:263-272.

5. Fowler TP, Marsh JL. Reduction and pinning of pediatric supracondylar humerus fractures in the prone position. $\mathbf{J}$ Orthop Trauma. 2006; 20:277-281.

6. Omid R, Choi PD, Skaggs DL. Supracondylar humeral fractures in children. J Bone Joint Surg Am. 2008; 90:1121-1132.

7. Parmaksizoglu AS, Ozkaya U, Bilgili F, Sayin E, Kabukcuoglu Y. Closed reduction of the pediatric supracondylar humerus fractures: the joystick method. Arch Orthop Trauma Surg. 2009; 129:1225-1231.

8. Pirone AM, Graham HK, Krajbich JI. Management of displaced extension-type supracondylar fractures of the humerus in children. J Bone Joint Surg Am. 1988; 70:641-650.

9. Skaggs DL, Flynn JM. Supracondylar fractures of the distal humerus In: Beaty JH, Kasser JR, eds. Rockwood and Green's Fractures in Children. 7th Ed. Philadelphia, PA: Lippincott Williams \& Wilkins, 2010, 487-532.

10. Smith L. Deformity following supracondylar fractures of the humerus. J Bone Joint Surg Am. 1960; 42:235-252.

11. Suh SW, Oh CW, Shingade VU, Swapnil MK, Park BC, Lee $\mathrm{SH}$ et al. Minimally invasive surgical techniques for irreducible supracondylar fractures of the humerus in children. Acta Orthop. 2005; 76:862-866.

12. Swenson AL. The treatment of supracondylar fractures of the humerus by Kirschner-wire transfixation. J Bone Joint Surg Am. 1948; 30:993-997. 